\title{
A Combinatorial Formula for the Hilbert Series of bigraded $S_{n}$-modules
}

\author{
Meesue Yoo \\ Department of Mathematics \\ University of California, San Diego, CA \\ meyoo@math.ucsd.edu \\ Submitted: Oct 20, 2009; Accepted: Jun 15, 2010; Published: Jun 29, 2010 \\ Mathematics Subject Classification: 05C88
}

\begin{abstract}
We prove a combinatorial formula for the Hilbert series of the Garsia-Haiman bigraded $S_{n}$-modules as weighted sums over standard Young tableaux in the hook shape case. This method is based on the combinatorial formula of Haglund, Haiman and Loehr for the Macdonald polynomials and extends the result of A. Garsia and C. Procesi for the Hilbert series when $q=0$. Moreover, we construct an association of the fillings giving the monomial terms of Macdonald polynomials with the standard Young tableaux.
\end{abstract}

\section{Introduction}

In 1988 [Mac88], Macdonald introduced a family of symmetric functions with two variables that are known as the Macdonald polynomials which form a basis for the space of symmetric functions. Upon introducing these polynomials, Macdonald conjectured that the coefficients of the plethystic Schur expansion of Macdonald polynomials are polynomials in the parameters $q$ and $t$ with nonnegative integer coefficients. To prove this positivity conjecture of Macdonald polynomials, Garsia and Haiman [GH93] introduced certain bigraded $S_{n}$ modules $M_{\mu}$ and Haiman proved [Hai01] that the bigraded Frobenius characteristic $\mathcal{F}\left(M_{\mu}\right)$, which by definition is simply the image of the bigraded character of $M_{\mu}$ under the Frobenius map, is given by

$$
\mathcal{F}_{M_{\mu}}(X ; q, t)=\tilde{H}_{\mu}(X ; q, t),
$$

where $\tilde{H}_{\mu}(X ; q, t)$ are the modified Macdonald polynomials [HHL05] and $X=x_{1}, x_{2}, \ldots$ For the Garsia-Haiman module $M_{\mu}$, if we define $\mathcal{H}_{h, k}\left(M_{\mu}\right)$ to be the subspace of $M_{\mu}$ 
spanned by its bihomogeneous elements of degree $h$ in $X$ and degree $k$ in $Y$, we can write a bivariate Hilbert series such as

$$
\mathcal{H}_{M_{\mu}}(q, t)=\sum_{h=0}^{n(\mu)} \sum_{k=0}^{n\left(\mu^{\prime}\right)} t^{h} q^{k} \operatorname{dim}\left(\mathcal{H}_{h, k}\left(M_{\mu}\right)\right)
$$

Noting that the degree of the $S_{n}$ character $\chi_{\lambda}$ is given by $\left\langle p_{1}^{n}, s_{\lambda}>\right.$, where $<,>$ is the usual inner product on symmetric functions and $p_{k}$ is the $k^{\text {th }}$ power sum, we may write

$$
\mathcal{H}_{M_{\mu}}(q, t)=<p_{1}^{n}, \mathcal{F}_{M_{\mu}}>
$$

Since $\mathcal{F}_{M_{\mu}}(X ; q, t)=\tilde{H}_{\mu}(X ; q, t)$, the coefficient of $x_{1} x_{2} \cdots x_{n}$ of $\tilde{H}_{\mu}(X ; q, t)$ gives the Hilbert series of Garsia-Haiman module $M_{\mu}$. In this paper, we construct a combinatorial way of calculating the Hilbert series of $M_{\mu}$ as a sum over all standard Young Tableaux with shape $\mu$ when $\mu$ is a hook.

We should mention that in the hook case, the Garsia-Haiman modules have been studied by Stembridge [Ste94], Garsia and Haiman [GH96], Allen [All02], Aval [Ava00], and Adin, Remmel and Roichman [ARR08] and various bases have been constructed. In 2004, Haglund, Haiman and Loehr proved a combinatorial formula for the monomial expansion of $\tilde{H}_{\mu}(X ; q, t)$ given by [HHL05]

$$
\tilde{H}_{\mu}(X ; q, t)=\sum_{\sigma: \mu \rightarrow \mathbb{Z}_{+}} q^{\operatorname{inv}(\mu, \sigma)} t^{\operatorname{maj}(\mu, \sigma)} x^{\sigma}
$$

where the definitions of $\operatorname{inv}(\mu, \sigma)$ and maj $(\mu, \sigma)$ are given in Section 3. The Hilbert series of $M_{\mu}$ can be easily calculated from the basis of the module or by the monomial expansion formula (1.1) of Haglund, Haiman and Loehr, but we have to consider $n$ ! many objects in any basis formula.

In this paper, we introduce a new combinatorial formula for this Hilbert series when $\mu$ is a hook shape which can be calculated by summing terms over only the standard Young tableaux of shape $\mu$. Noting that the number of SYT's of shape $\mu$ is $n ! / \prod_{c \in \mu} h(c)$ where $h(c)=a(c)+l(c)+1$, obviously this combinatorial formula reduces the number of objects that we need to consider to calculate the Hilbert series. This combinatorial formula is motivated by the formula for the two-column shape case which is conjectured by Haglund and proved by Garsia and Haglund [GH08]. Assaf and Garsia [AG09] used the recursion derived by the combinatorial formula for the two-column case to find the kicking basis of $M_{\mu}$, and extended the result to find the kicking basis when $\mu$ has a hook shape. In Section 5, we also introduce a way of finding the Haglund basis [ARR08] by using the combinatorial construction of the hook case.

The outline of this paper is as follows. In Section 2, we define terms that are used in this paper and introduce what Macdonald polynomials and Garsia-Haiman modules are. In Section 3, we construct a combinatorial formula and prove it. In Section 4, we find the correspondence between the terms in the formula of Haglund, Haiman and Loehr and the combinatorial formula in Section 3. In Section 5, we find the basis of Garsia-Haiman 
modules by using the combinatorial construction and the correspondence introduced in Section 4. In Section 6, we discuss the problem of extending the combinatorial formula to general shapes.

\section{Macdonald Polynomials and Bigraded $S_{n}$ Modules}

Given a sequence $\mu=\left(\mu_{1}, \mu_{2}, \ldots\right)$ of nonincreasing, nonnegative integers with $\sum_{i} \mu_{i}=n$, we say $\mu$ is a partition of $n$, denoted by either $|\mu|=n$ or $\mu \vdash n$. Let

$$
\operatorname{dg}(\mu)=\left\{(i, j) \in \mathbb{Z}_{+} \times \mathbb{Z}_{+}: j \leqslant \mu_{i}\right\}
$$

be its Young (or Ferrers) diagram, whose elements are called cells. For simplicity, we henceforth write $\mu$ instead of $\operatorname{dg}(\mu)$ when it will not cause confusion.

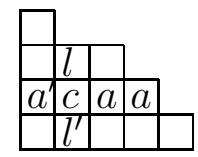

Figure 1: The $\operatorname{arm} a, \operatorname{leg} l, \operatorname{coarm} a^{\prime}$ and coleg $l^{\prime}$ of a cell $c$.

Given a square $c \in \mu$, define the leg (respectively coleg) of $c$, denoted $l(c)\left(\right.$ resp. $l^{\prime}(c)$ ), to be the number of squares in $\mu$ that are strictly above (resp. below) and in the same column as $c$, and the arm (resp. coarm) of $c$, denoted $a(c)$ (resp. $a^{\prime}(c)$ ), to be the number of squares in $\mu$ strictly to the right (resp. left) and in the same row as $c$. Also, if $c$ has coordinates $(i, j)$, we let $\operatorname{south}(c)$ denote the square with coordinates $(i-1, j)$.

For each partition $\mu$ define

$$
n(\mu)=\sum_{i \geqslant 1}(i-1) \mu_{i}
$$

A filling is a function $\sigma: \mu \rightarrow[n]$ assigning integer entries to the cells of $\mu$. A semistandard Young tableau is a filling which is weakly increasing along each row of $\mu$ and strictly increasing up each column. A semi-standard Young tableau is standard if it is a bijection from $\mu$ to $[n]=\{1,2, \ldots, n\}$. For a partition $\mu$ of $n$ and a composition $\nu$ of $n$, we define

$$
\begin{aligned}
& \operatorname{SSYT}(\mu)=\{\text { semi-standard Young tableau } T: \mu \rightarrow \mathbb{N}\} \text {, } \\
& \operatorname{SSYT}(\mu, \nu)=\left\{\operatorname{SSYT} T: \mu \rightarrow \mathbb{N} \text { with entries } 1^{\nu_{1}}, 2^{\nu_{2}}, \ldots\right\} \text {, } \\
& \operatorname{SYT}(\mu)=\{\operatorname{SSYT} T: \mu \stackrel{\sim}{\rightarrow}[n]\}=\operatorname{SSYT}\left(\mu, 1^{n}\right) \text {. }
\end{aligned}
$$

For $T \in \operatorname{SSYT}(\mu, \nu)$, we say $T$ is a SSYT of shape $\mu$ and weight $\nu$. 


\subsection{Macdonald Polynomials}

In 1988, Macdonald [Mac95] introduced a new basis of symmetric functions, denoted by $P_{\mu}(X ; q, t), X=x_{1}, x_{2}, \ldots$, which specializes to Schur functions, the Hall-Littlewood symmetric functions, the Jack symmetric functions, the zonal symmetric functions, and the elementary and monomial symmetric functions. With an appropriate analog of the Hall inner product, $P_{\mu}(X ; q, t)$ are uniquely characterized by certain triangularity and orthogonality conditions. For each partition $\mu$, define

$$
h_{\mu}(q, t):=\prod_{c \in \mu}\left(1-q^{a(c)} t^{l(c)+1}\right) .
$$

Macdonald introduced the $q, t$-Kostka polynomials $K_{\lambda \mu}(q, t)$ by the equation

$$
J_{\mu}(X ; q, t)=h_{\mu}(q, t) P_{\mu}(X ; q, t)=\sum_{\lambda} K_{\lambda \mu}(q, t) s_{\lambda}[X(1-t)],
$$

where the square bracket stands for plethystic substitution. In short, $s_{\lambda}[A]$ means $s_{\lambda}$ applied as a $\Lambda$-ring operator to the expression $A$, where $\Lambda$ is the ring of symmetric functions. For a full account of plethysm, see [Hai99]. In attempt to prove the positivity conjecture, Garsia and Haiman [GH93] introduced the modified Macdonald polynomials $\tilde{H}_{\mu}(X ; q, t)$ as

$$
\tilde{H}_{\mu}(X ; q, t)=\sum_{\lambda} \tilde{K}_{\lambda \mu}(q, t) s_{\lambda}[X],
$$

where $\tilde{K}_{\lambda \mu}(q, t):=t^{n(\mu)} K_{\lambda \mu}\left(q, t^{-1}\right)$. They conjectured [GH93] that $\tilde{H}_{\mu}(X ; q, t)$ can be realized as the Frobenius image of bigraded character of certain modules $M_{\mu}$ under the diagonal action of $S_{n}$. This is known as the $n$ ! conjecture, and by analyzing the algebraic geometry of the Hilbert series of $n$ points in the plane, Haiman [Hai01] proved the $n$ ! conjecture and consequently the Macdonald positivity conjecture.

\subsection{Garsia-Haiman Modules}

Let $\mu$ be a partition and let $\left(p_{1}, q_{1}\right), \ldots,\left(p_{n}, q_{n}\right)$ denote the pairs $\left(l^{\prime}(c), a^{\prime}(c)\right)$ of the cells $c$ of the diagram of $\mu$ arranged in lexicographic order. We set

$$
\triangle_{\mu}(X, Y)=\triangle_{\mu}\left(x_{1}, \ldots, x_{n} ; y_{1}, \ldots, y_{n}\right)=\operatorname{det}\left\|x_{i}^{p_{j}} y_{i}^{q_{j}}\right\|_{i, j=1, \ldots, n} .
$$

Example 2.1. For $\mu=(3,1),\left\{\left(p_{j}, q_{j}\right)\right\}=\{(0,0),(0,1),(0,2),(1,0)\}$, and

$$
\triangle_{\mu}=\operatorname{det}\left(\begin{array}{cccc}
1 & y_{1} & y_{1}^{2} & x_{1} \\
1 & y_{2} & y_{2}^{2} & x_{2} \\
1 & y_{3} & y_{3}^{2} & x_{3} \\
1 & y_{4} & y_{4}^{2} & x_{4}
\end{array}\right)
$$


This given, we let $M_{\mu}[X, Y]$ be the space spanned by all the partial derivatives of $\triangle_{\mu}(x, y)$. In symbols

$$
M_{\mu}[X, Y]=\mathcal{L}\left[\partial_{x}^{p} \partial_{y}^{q} \triangle \mu(x, y)\right]
$$

where $\partial_{x}^{p}=\partial_{x_{1}}^{p_{1}} \cdots \partial_{x_{n}}^{p_{n}}, \partial_{y}^{p}=\partial_{y_{1}}^{p_{1}} \cdots \partial_{y_{n}}^{p_{n}}$. The diagonal action of a permutation $\sigma=$ $\left(\sigma_{1}, \ldots, \sigma_{n}\right)$ on a polynomial $P\left(x_{1}, \ldots, x_{n} ; y_{1}, \ldots, y_{n}\right)$ is defined by setting

$$
\sigma P\left(x_{1}, \ldots, x_{n} ; y_{1}, \ldots, y_{n}\right):=P\left(x_{\sigma(1)}, \ldots, x_{\sigma(n)} ; y_{\sigma(1)}, \ldots, y_{\sigma(n)}\right) .
$$

Since $\sigma \triangle_{\mu}= \pm \triangle_{\mu}$ according to the sign of $\sigma$, the space $M_{\mu}$ necessarily remains invariant under this action.

Note that, since $\triangle_{\mu}$ is bihomogeneous of degree $n(\mu)$ in $x$ and $n\left(\mu^{\prime}\right)$ in $y$, we have the direct sum decomposition

$$
M_{\mu}=\oplus_{h=0}^{n(\mu)} \oplus_{k=0}^{n\left(\mu^{\prime}\right)} \mathcal{H}_{h, k}\left(M_{\mu}\right),
$$

where $\mathcal{H}_{h, k}\left(M_{\mu}\right)$ denotes the subspace of $M_{\mu}$ spanned by its bihomogeneous elements of degree $h$ in $x$ and degree $k$ in $y$. Since the diagonal action clearly preserves bidegree, each of the subspaces $\mathcal{H}_{h, k}\left(M_{\mu}\right)$ is also $S_{n}$-invariant. Thus we see that $M_{\mu}$ has the structure of a bigraded module. We can write a bivariate Hilbert series such as

$$
F_{\mu}(q, t)=\sum_{h=0}^{n(\mu)} \sum_{k=0}^{n\left(\mu^{\prime}\right)} t^{h} q^{k} \operatorname{dim}\left(\mathcal{H}_{h, k}\left(M_{\mu}\right)\right) .
$$

In 2001, Haiman [Hai01] proved that the bigraded character of $M_{\mu}$ is given by

$$
\mathcal{F}_{M_{\mu}}(X ; q, t)=\sum_{h=0}^{n(\mu)} \sum_{k=0}^{n\left(\mu^{\prime}\right)} t^{h} q^{k} \psi\left(\mathcal{H}_{h, k}\left(M_{\mu}\right)\right)=\tilde{H}_{\mu}(X ; q, t)
$$

where $\psi$ is the Frobenius map sending the Specht module $S^{\lambda}$ to the Schur function $s_{\lambda}$. Then the Hilbert series can be calculated by using the monomial expansion formula (1.1) as a sum over $n$ ! permutations of $n$ numbers, that is

$$
F_{\mu}(q, t)=\sum_{\sigma \in S_{n}} q^{\operatorname{inv}(\mu, \sigma)} t^{\operatorname{maj}(\mu, \sigma)} .
$$

For the definitions of $\operatorname{inv}(\mu, \sigma)$ and $\operatorname{maj}(\mu, \sigma)$, see Section 3.

\subsection{Macdonald's Construction}

The combinatorial construction is based on the following fact known by Macdonald [Mac95] and noticed by Haglund [Hag]. Upon the introduction of Macdonald polynomials [Mac88], Macdonald defined another family of symmetric functions $\left\{Q_{\mu}(X ; q, t)\right\}$ by

$$
Q_{\mu}(X ; q, t)=\frac{h_{\mu}^{\prime}(q, t)}{h_{\mu}(q, t)} P_{\mu}(X ; q, t)
$$


where $h_{\mu}(q, t):=\prod_{c \in \mu}\left(1-q^{a(c)} t^{l(c)+1}\right)$ and $h_{\mu}^{\prime}(q, t):=\prod_{c \in \mu}\left(1-q^{a(c)+1} t^{l(c)}\right)$, and so

$$
J_{\mu}(X ; q, t)=h_{\mu}(q, t) P_{\mu}(X ; q, t)=h_{\mu}^{\prime}(q, t) Q_{\mu}(X ; q, t) .
$$

Noting that $P_{\mu}(X ; 0, t)=P_{\mu}(X ; t)$ are the Hall-Littlewood polynomials, there are corresponding symmetric functions $Q_{\mu}(X ; 0, t)=Q_{\mu}(X ; t)$ which can be independently defined by

$$
Q_{\mu}(X ; t)=b_{\mu}(t) P_{\mu}(X ; t)
$$

where

$$
b_{\mu}(t)=\prod_{i \geqslant 1} \varphi_{m_{i}(\mu)}(t)
$$

and $m_{i}(\mu)$ denotes the number of times $i$ occurs as a part of $\mu$ and $\varphi_{r}(t)=(1-t)(1-$ $\left.t^{2}\right) \cdots\left(1-t^{r}\right)$. In [Mac95, Ch. III, (5.11)], Macdonald proved the following. Let $T$ be a semistandard tableau of shape $\mu$ and weight $\nu$. Then $T$ determines a sequence of partitions $\left(\mu^{(0)}, \ldots, \mu^{(r)}\right)$ such that $0=\mu^{(0)} \subset \mu^{(1)} \subset \cdots \subset \mu^{(r)}=\mu$ and such that each $\mu^{(i)}-\mu^{(i-1)}$ is a horizontal strip filled with $i$. Let

$$
\varphi_{T}(t)=\prod_{i=1}^{r} \varphi_{\mu^{(i)} / \mu^{(i-1)}}(t)
$$

then

$$
Q_{\mu}(X ; t)=\sum_{T \in \operatorname{SSYT}(\mu)} \varphi_{T}(t) x^{T} .
$$

Then the Macdonald polynomials

$$
H_{\mu}(X ; q, t)=J_{\mu}\left[\frac{X}{1-t} ; q, t\right]=\sum_{\lambda} K_{\lambda \mu}(q, t) s_{\lambda}[X]
$$

satisfy

$$
H_{\mu}(X ; 0, t)=\frac{1}{(1-t)^{n}} \sum_{T \in \operatorname{SSYT}(\mu)} \varphi_{T}(t) x^{T}
$$

when $q=0$, and the Hilbert series become

$$
\tilde{F}_{\mu^{\prime}}(0, t)=\frac{1}{(1-t)^{n}} \sum_{T \in \operatorname{SYT}(\mu)} \varphi_{T}(t) .
$$

This gives a combinatorial construction of the $t$ factor of the Hilbert series of GarsiaHaiman modules when $q=0$. This is true for any general shape of $\mu$. Based on (2.3), the combinatorial formula for the Hilbert series for the two column case was constructed by Garsia and Haglund [GH08]. We consider a hook case in this paper. 


\subsection{Two Column Case}

Garsia and Haglund [GH08] proved that when $\mu=\left(2^{b}, 1^{a-b}\right)$, the Hilbert series $F_{\mu}(q, t)$ has the combinatorial formula

$$
F_{\mu}(q, t)=\sum_{T \in \operatorname{SYT}(\mu)} \prod_{i \in T}\left[d_{i}(T)\right]_{t} \prod_{\substack{i \text { in the second } \\ \text { column of } T}}\left(q+t^{b_{i}(T)}\right)
$$

where the sum is over all standard Young tableaux of shape $\mu, d_{i}(T)$ is the number of rows of length equal to the length of the row of $i$ in the tableau obtained by removing all the entries $j>i$ from $T$, the second product is over entries in the second column of $T$, and $b_{i}(T)$ denotes the number of entries $j>i$ in the first column of $T$. This combinatorial construction gives the following recursion of $F_{\mu}(q, t)$

$$
F_{2^{b} 1^{a-b}}(q, t)=[b]_{t}(1+q) F_{2^{b-1} 1^{a-b+1}}(q, t)+[a-b]_{t} t^{b} F_{2^{b} 1^{a-b-1}}(q / t, t)
$$

and since $F_{\mu}(q, t)=\partial_{p_{1}}^{n} \tilde{H}_{\mu}[X ; q, t]$, this recursion suggests the Frobenius characteristic recursion

$$
\partial_{p_{1}} \tilde{H}_{2^{b} 1^{a-b}}(q, t)=[b]_{t}(1+q) \tilde{H}_{2^{b-1} 1^{a-b+1}}(q, t)+[a-b]_{t} t^{b} \tilde{H}_{2^{b} 1^{a-b-1}}(q / t, t) .
$$

Assaf and Garsia [AG09] applied (2.4) to find the kicking basis of $M_{\mu}$ when $\mu$ is a column shape as well as when $\mu$ is a hook shape.

\section{The Formula}

We begin by recalling definitions of $q$-analogs :

$$
\begin{aligned}
& {[n]_{q}=1+q+\cdots+q^{n-1},} \\
& {[n]_{q} !=[1]_{q} \cdots[n]_{q} .}
\end{aligned}
$$

A descent of a filling $\sigma$ of $\mu$ is a pair of entries $\sigma(u)>\sigma(v)$, where the cell $u$ is immediately above $v$. Define

$$
\operatorname{Des}(\sigma, \mu)=\{u \in \mu: \sigma(u)>\sigma(v) \text { a descent }\},
$$

and

$$
\operatorname{maj}(\sigma, \mu)=\sum_{u \in \operatorname{Des}(\sigma, \mu)}(\operatorname{leg}(u)+1) .
$$

Three cells $u, v, w \in \mu$ are said to form a triple if they are situated as shown below.

\begin{tabular}{|l|l|}
\hline $\mathrm{u}$ & $\mathrm{w}$ \\
\hline $\mathrm{v}$ &
\end{tabular}


namely, $v$ is directly below $u$, and $w$ is in the same row as $u$, to its right. Let $\sigma$ be a filling and let $x, y, z$ be the entries of $\sigma$ in the cells of a triple $(u, v, w)$.

\begin{tabular}{|l|l|}
\hline $\mathrm{y}$ & $\mathrm{Z}$ \\
$\mathrm{x}$ &
\end{tabular}

If a path starting from the smallest entry to the largest entry rotates in a counter clockwise way, then the triple is called an inversion triple. Otherwise, it is called a coinversion triple. Define

$$
\begin{gathered}
\operatorname{inv}(\sigma, \mu)=\text { number of inversion triples of } \sigma, \\
\operatorname{coinv}(\sigma, \mu)=\text { number of coinversion triples of } \sigma .
\end{gathered}
$$

For convenience, we make a transformation to define $\tilde{F}_{\mu^{\prime}}(q, t)$ by

$$
\tilde{F}_{\mu^{\prime}}(q, t)=t^{n(\mu)} F_{\mu}\left(\frac{1}{t}, q\right)
$$

where $n(\mu)=\sum_{i \geqslant 1}(i-1) \mu_{i}$. We note that by modifying the $\operatorname{inv}(\mu, \sigma)$ statistics, we get

$$
\tilde{F}_{\mu^{\prime}}(q, t)=\sum_{\sigma \in S_{n}} q^{\operatorname{maj}\left(\sigma, \mu^{\prime}\right)} t^{\operatorname{coinv}\left(\sigma, \mu^{\prime}\right)}
$$

Now, for a hook $\mu^{\prime}=\left(n-s, 1^{s}\right)$, we define a combinatorial formula for the Hilbert series as a sum over standard Young tableaux of shape $\mu^{\prime}$ by setting

$$
G_{\mu}(q, t)=q^{n(\mu)} \tilde{G}_{\mu^{\prime}}\left(t, \frac{1}{q}\right)
$$

where $\tilde{G}_{\mu^{\prime}}(q, t)$ is defined by

$$
\tilde{G}_{\mu^{\prime}}(q, t):=\sum_{T \in \operatorname{SYT}\left(\mu^{\prime}\right)} \prod_{i=1}^{n}\left[a_{i}(T)\right]_{t} \cdot[s]_{q} !\left(1+\sum_{j=1}^{s} q^{j} t^{b_{j}(T)}\right) .
$$

Here $a_{i}(T)$ is the the number of columns of height equal to the height of the column of $i$ in the tableau obtained by removing all the entries $j>i$ from $T$, and $b_{j}(T)$ is the number of cells in the first row with column height 1 (i.e., strictly to the right of the $(1,1)$ cell) with bigger element than the element in the $(s-j+2,1)$ cell. Then we have the following theorem :

Theorem 3.1.

$$
\tilde{F}_{\left(n-s, 1^{s}\right)^{\prime}}(q, t)=\tilde{G}_{\left(n-s, 1^{s}\right)^{\prime}}(q, t),
$$

and so

$$
F_{\left(s+1,1^{n-s-1}\right)}(q, t)=G_{\left(s+1,1^{n-s-1}\right)}(q, t) .
$$


Example 3.2. Let $\mu=(2,1)$. To calculate $\tilde{F}_{(2,1)}(q, t)=\sum_{\sigma \in S_{3}} q^{\operatorname{maj}\left(\sigma, \mu^{\prime}\right)} t^{\operatorname{coinv}\left(\sigma, \mu^{\prime}\right)}$, we must consider the following tableaux.

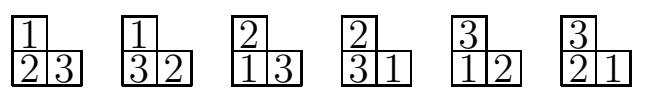

From the above tableaux, reading from the left, we get

$$
\tilde{F}_{(2,1)}(q, t)=t+1+q t+1+q t+q=2+q+t+2 q t .
$$

To compute $\tilde{G}_{(2,1)}(q, t)$, we need only consider the following two standard tableaux.

$$
T_{1}=\begin{array}{ll}
2 & 3
\end{array}, \quad T_{2}=\begin{array}{ll}
3 \\
\hline 12
\end{array}
$$

We calculate $a_{i}\left(T_{k}\right), b_{j}\left(T_{k}\right)$, for $1 \leqslant i \leqslant 3, j=1, k=1,2$ :

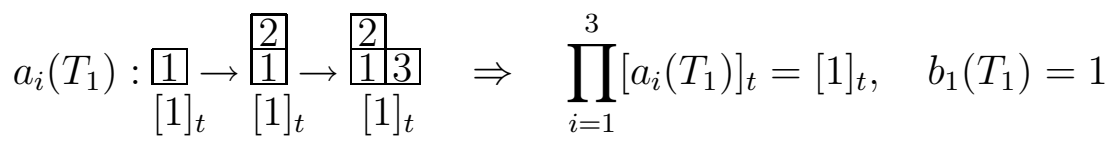

$$
\begin{aligned}
& \Rightarrow[1]_{t} \cdot(1+q t) \\
& a_{i}\left(T_{2}\right): \underset{[1]_{t}}{: \frac{1 \mid 2}{[2]_{t}}} \rightarrow \frac{\frac{3}{1 / 2}}{[1]_{t}} \Rightarrow \prod_{i=1}^{3}\left[a_{i}\left(T_{2}\right)\right]_{t}=[2]_{t}, \quad b_{1}\left(T_{2}\right)=0 \\
& \Rightarrow(1+t) \cdot(1+q) \text {. }
\end{aligned}
$$

So,

$$
\begin{aligned}
\tilde{G}_{(2,1)}(q, t) & =1 \cdot(1+q t)+(1+t)(1+q) \\
& =2+q+t+2 q t .
\end{aligned}
$$

We can check that $\tilde{F}_{(2,1)}(q, t)=\tilde{G}_{(2,1)}(q, t)$ which implies $F_{(2,1)}(q, t)=G_{(2,1)}(q, t)$.

The basic idea of the proof of Theorem 3.1 is to show $F_{\mu}(q, t)$ and $G_{\mu}(q, t)$ satisfy the same recursion in the hook case.

Proof. We first note the Garsia-Haiman recursion for the Hilbert series of the hooks [GH96] : for $\mu=\left(s+1,1^{n-s-1}\right)$,

$$
\begin{gathered}
F_{\mu}(q, t)=[n-s-1]_{t} F_{\left(s+1,1^{n-s-2}\right)}(q, t)+\left(\begin{array}{c}
n-1 \\
s
\end{array}\right) t^{n-s-1}[n-s-1]_{t} ![s]_{q} ! \\
+q[s]_{q} F_{\left(s, 1^{n-s-1}\right)}(q, t) .
\end{gathered}
$$

We derive the recursion formula for $\tilde{G}_{\mu^{\prime}}(q, t)$ over standard tableaux by fixing the position of the cell with the largest number $n$ :
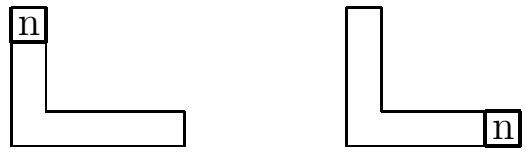
Let's first start from a SYT of shape $\left(n-s, 1^{s-1}\right)$ and say

$$
\tilde{G}_{\left(n-s, 1^{s-1}\right)}(q, t)=\sum_{T \in \operatorname{SYT}\left(\left(n-s, 1^{s-1}\right)\right)} \prod_{i=1}^{n-1}\left[a_{i}(T)\right]_{t} \cdot[s-1]_{q} !\left(1+\sum_{j=1}^{s-1} q^{j} t^{b_{j}(T)}\right)
$$

and put the cell with $n$ on the top of the first column. Then, since there is no other column with height $s+1$, adding the cell with $n$ on the top of the first column gives $a_{n}(T)=1$ which doesn't change the first factor of (3.4). The change of the first column height from $s-1$ to $s$ will give an additional factor $[s]_{q}$. The top cell in the first column with $n$ has $t$ power 0 since $n$ is the largest number, and it does not change $t$-statistics for the cells below the cell with $n$, so $\left(1+\sum_{j=1}^{s-1} q^{j} t^{b_{j}(T)}\right)$ changes to $\left(1+q+\sum_{j=2}^{s} q^{j} t^{b_{j-1}(T)}\right)$. Hence, for the first tableau with $n$ on the top of the first column, the formula becomes

$$
\begin{aligned}
\sum_{T \in \operatorname{SYT}\left(\left(n-s, 1^{s-1}\right)\right)} & \prod_{i=1}^{n-1}\left[a_{i}(T)\right]_{t} \cdot[s]_{q} !\left[1+q\left(1+\sum_{j=1}^{s-1} q^{j} t^{b_{j}(T)}\right)\right] \\
& =\left(\sum_{T \in \operatorname{SYT}\left(n-s, 1^{s-1}\right)} \prod_{i=1}^{n-1}\left[a_{i}(T)\right]_{t} \cdot[s]_{q} !\right)+q[s]_{q} \tilde{G}_{\left(n-s, 1^{s-1}\right)}(q, t)
\end{aligned}
$$

and in terms of $\tilde{G}_{\left(n-s, 1^{s-1}\right)}(q, t)$, this is equal to

$$
[s]_{q} ! \tilde{G}_{\left(n-s, 1^{s-1}\right)}(0, t)+q[s]_{q} \tilde{G}_{\left(n-s, 1^{s-1}\right)}(q, t) .
$$

In the second tableau case, we start from a SYT of shape $\left(n-s-1,1^{s}\right)$ and add the cell with $n$ to the end of the first row. Adding a cell with $n$ to the end of the first row increases the number of columns with height 1 from $n-s-2$ to $n-s-1$, so it contributes the $t$ factor $\left[a_{n}(T)\right]_{t}=[n-s-1]_{t}$. Since it doesn't affect the first column, $[s]_{q}$ ! stays, but having the largest number $n$ in the first row increases all the $b_{j}(T)$ 's by 1 . In other words, if we let the formula for the SYT of shape $\left(n-s-1,1^{s}\right)$ be

$$
\tilde{G}_{\left(n-s-1,1^{s}\right)}(q, t)=\sum_{T \in \operatorname{SYT}\left(\left(n-s-1,1^{s}\right)\right)} \prod_{i=1}^{n-1}\left[a_{i}(T)\right]_{t} \cdot[s]_{q} !\left(1+\sum_{j=1}^{s} q^{j} t^{b_{j}(T)}\right)
$$

then by adding the cell with $n$ in the end of the first row, it changes to

$$
\begin{aligned}
& \sum_{T \in \operatorname{SYT}\left(n-s-1,1^{s}\right)}[n-s-1]_{t} \cdot \prod_{i=1}^{n-1}\left[a_{i}(T)\right]_{t} \cdot[s]_{q} !\left(1+\sum_{j=1}^{s} q^{j} t^{b_{j}(T)+1}\right) \\
= & \sum_{T \in \operatorname{SYT}\left(n-s-1,1^{s}\right)}[n-s-1]_{t} \cdot \prod_{i=1}^{n-1}\left[a_{i}(T)\right]_{t} \cdot[s]_{q} !\left[t\left(1+\sum_{j=1}^{s} q^{j} t^{b_{j}(T)}\right)+(1-t)\right] .
\end{aligned}
$$


In terms of $\tilde{G}_{\left(n-s-1,1^{s}\right)}$, this can be expressed as

$$
t[n-s-1]_{t} \tilde{G}_{\left(n-s-1,1^{s}\right)}(q, t)+(1-t)[n-s-1]_{t}[s]_{q} ! \tilde{G}_{\left(n-s-1,1^{s}\right)}(0, t) .
$$

By adding (3.5) and (3.6), we get the following recursive formula for $\tilde{G}_{\left(n-s, 1^{s}\right)}(q, t)$ :

$$
\begin{aligned}
\tilde{G}_{\left(n-s, 1^{s}\right)}(q, t)= & q[s]_{q} \tilde{G}_{\left(n-s, 1^{s-1}\right)}(q, t)+t[n-s-1]_{t} \tilde{G}_{\left(n-s-1,1^{s}\right)}(q, t) \\
& +[s]_{q} !\left(\tilde{G}_{\left(n-s, 1^{s-1}\right)}(0, t)+\left(1-t^{n-s-1}\right) \tilde{G}_{\left(n-s-1,1^{s}\right)}(0, t)\right) .
\end{aligned}
$$

To compare this to the recursion of $F_{\mu}(q, t)(3.3)$, we do the transformation $G_{\mu}(q, t)=$ $q^{n(\mu)} \tilde{G}_{\mu^{\prime}}\left(t, \frac{1}{q}\right)$ and we get the recursion formula for $G_{\mu}(q, t)$

$$
\begin{aligned}
& G_{\left(s+1,1^{n-s-1}\right)}(q, t)=q[s]_{q} G_{\left(s, 1^{n-s-1}\right)}(q, t)+[n-s-1]_{t} G_{\left(s+1,1^{n-s-2}\right)}(q, t) \\
&+[s]_{q} !\left\{G_{\left(s, 1^{n-s-1}\right)}(0, t)+\left(t^{n-s-1}-1\right) G_{\left(s+1,1^{n-s-2}\right)}(0, t)\right\} .
\end{aligned}
$$

We calculate the last two terms in (3.7).

\section{Lemma 3.3.}

$$
[s]_{q} !\left\{G_{\left(s, 1^{n-s-1}\right)}(0, t)+\left(t^{n-s-1}-1\right) G_{\left(s+1,1^{n-s-2}\right)}(0, t)\right\}=[n-s-1]_{t} ![s]_{q} !\left(\begin{array}{c}
n-1 \\
s
\end{array}\right) t^{n-s-1} .
$$

Proof. We use (3.4) and do the transformation $G_{\mu}(q, t)=q^{n(\mu)} \tilde{G}_{\mu^{\prime}}\left(t, \frac{1}{q}\right)$ to calculate $G_{\left(s, 1^{n-s-1}\right)}(0, t)$ and $G_{\left(s+1,1^{n-s-2}\right)}(0, t)$ when $q=0$. We have

$$
\begin{aligned}
& G_{\left(s, 1^{n-s-1}\right)}(q, t)=[n-s-1]_{t} ! t^{n-s-1}[s-1]_{q} ! \\
& \quad \times \sum_{j_{1}=2}^{n-s+1} \cdots \sum_{j_{s-1}=j_{s-2}+1}^{n-1} \frac{\left[j_{1}-1\right]_{t}}{t^{j_{1}-2}}\left(1+q t^{-\left(n-s-j_{s-1}\right)}+\cdots+q^{s-1} t^{-\left(n-1-j_{1}-(s-2)\right)}\right) .
\end{aligned}
$$

Then for $G_{\left(s, 1^{n-s-1}\right)}(0, t)$, we get

$$
\begin{aligned}
G_{\left(s, 1^{n-s-1}\right)}(0, t) & =[n-s-1]_{t} ! t^{n-s-1} \sum_{j_{1}=2}^{n-s+1} \cdots \sum_{j_{s-1}=j_{s-2}+1}^{n-1} \frac{\left[j_{1}-1\right]_{t}}{t^{j_{1}-2}} \\
& =[n-s-1]_{t} ! t^{n-s-1} \sum_{j_{1}=2}^{n-s+1} \frac{\left[j_{1}-1\right]_{t}}{t^{j_{1}-2}}\left(\sum_{j_{2}=j_{1}+1}^{n-s+2} \cdots \sum_{j_{s-1}=j_{s-2}+1}^{n-1} 1\right) \\
& =[n-s-1]_{t} ! t^{n-s-1} \sum_{j_{1}=2}^{n-s+1} \frac{\left[j_{1}-1\right]_{t}}{t^{j_{1}-2}}\left(\begin{array}{c}
n-s-j_{1} \\
s-2
\end{array}\right) \\
& =[n-s-1]_{t} ! t^{n-s-1} \sum_{i=s}^{n-1}\left(\begin{array}{c}
i-1 \\
s-1
\end{array}\right) t^{-(n-1-i)} \\
& =[n-s-1]_{t} ! \sum_{i=s}^{n-1}\left(\begin{array}{c}
i-1 \\
s-1
\end{array}\right) t^{i-s} .
\end{aligned}
$$


Similarly, we get

$$
G_{\left(s+1,1^{n-s-2}\right)}(0, t)=[n-s-2] t ! \sum_{i=s+1}^{n-1}\left(\begin{array}{c}
i-1 \\
s
\end{array}\right) t^{i-s-1} .
$$

Hence,

$$
\begin{aligned}
& {[s]_{q} !\left\{G_{\left(s, 1^{n-s-1}\right)}(0, t)+\left(t^{n-s-1}-1\right) G_{\left(s+1,1^{n-s-2}\right)}(0, t)\right\}} \\
& =[s]_{q} !\left\{[n-s-1]_{t} ! \sum_{i=s}^{n-1}\left(\begin{array}{c}
i-1 \\
s-1
\end{array}\right) t^{i-s}+\left(t^{n-s-1}-1\right)[n-s-2]_{t} ! \sum_{i=s+1}^{n-1}\left(\begin{array}{c}
i-1 \\
s
\end{array}\right) t^{i-s-1}\right\} \\
& =[s]_{q} ![n-s-1]_{t} !\left\{\sum_{i=s}^{n-1}\left(\begin{array}{c}
i-1 \\
s-1
\end{array}\right) t^{i-s}+(t-1) \sum_{i=s+1}^{n-1}\left(\begin{array}{c}
i-1 \\
s
\end{array}\right) t^{i-s-1}\right\} \\
& =[s]_{q} ![n-s-1]_{t} !\left\{\sum_{i=s}^{n-1}\left(\begin{array}{c}
i-1 \\
s-1
\end{array}\right) t^{i-s}+\sum_{i=s+1}^{n-1}\left(\begin{array}{c}
i-1 \\
s
\end{array}\right) t^{i-s}-\sum_{i=s+1}^{n-1}\left(\begin{array}{c}
i-1 \\
s
\end{array}\right) t^{i-s-1}\right\} \\
& =[s]_{q} ![n-s-1]_{t} !\left(\begin{array}{c}
n-1 \\
s
\end{array}\right) t^{n-s-1} .
\end{aligned}
$$

Thus the recursion formula for $G_{\mu}(q, t)$ simplifies to

$$
\begin{gathered}
G_{\left(s+1,1^{n-s-1}\right)}(q, t)=q[s]_{q} G_{\left(s, 1^{n-s-1}\right)}(q, t)+[n-s-1]_{t} G_{\left(s+1,1^{n-s-2}\right)}(q, t) \\
+[n-s-1]_{t} ![s]_{q} !\left(\begin{array}{c}
n-1 \\
s
\end{array}\right) t^{n-s-1}
\end{gathered}
$$

We compare two recursions (3.3) and (3.8), and confirm that $F_{\mu}(q, t)$ and $G_{\mu}(q, t)$ both satisfy the same recursion. Based on the fact that $F_{(1)}(q, t)=G_{(1)}(q, t)$, we conclude that $F_{\mu}(q, t)=G_{\mu}(q, t)$, which also implies $\tilde{F}_{\mu^{\prime}}(q, t)=\tilde{G}_{\mu^{\prime}}(q, t)$. This finishes the proof.

Remark 3.4. We can define a combinatorial construction for $G_{\mu}(q, t)$ directly

$$
G_{\mu}(q, t)=\sum_{T \in \operatorname{SYT}(\mu)} \prod_{i=1}^{n}\left[a_{i}(T)\right]_{t}\left[\mu_{1}-1\right]_{q} !\left(\sum_{j=1}^{\mu_{1}-1} q^{j-1} t^{b_{j}(T)}+q^{\mu_{1}-1}\right)
$$

where $a_{i}(T)$ is the number of rows of length equal to the length of the row of $i$ in the tableau obtained by removing all the entries $j>i$ from $T$, and $b_{j}(T)$ counts the number of cells in the first column in rows strictly above the cell $(1,1)$ with bigger numbers than the element in the cell $(1, j+1)$.

The main reason for applying the modification $\tilde{G}_{\mu^{\prime}}(q, t)=t^{n(\mu)} G_{\mu}\left(\frac{1}{t}, q\right)$ is because the combinatorial construction for $G_{\mu}(q, t)$ does not give the recursion from the construction directly.

Remark 3.5. This factorization form in the hook case was independently noticed by Morita [Mor08, Proposition 13]. 


\section{Association with the Fillings}

In this section, we find the correspondence between a group of fillings and one standard Young tableau giving the same polynomial for the Hilbert series. In other words, we construct a way of grouping permutations $\left\{\sigma_{1}, \ldots, \sigma_{k}\right\}, \sigma_{i} \in S_{n}$, so that

$$
\sum_{i=1}^{k} q^{\operatorname{inv}\left(\mu, \sigma_{i}\right)} t^{\operatorname{maj}\left(\mu, \sigma_{i}\right)}=\prod_{i=1}^{n}\left[a_{i}(T)\right]_{t}\left[\mu_{1}-1\right]_{q} !\left(\sum_{j=1}^{\mu_{1}-1} q^{j-1} t^{b_{j}(T)}+q^{\mu_{1}-1}\right)
$$

where $T$ is a standard Young tableau of shape $\mu$ and the right hand side is the polynomial summed over $\operatorname{SYT}(\mu)$ in $(3.9)$.

In Section 4.1, we introduce a grouping table as a way to construct a grouping of fillings and we modify the Garsia-Procesi tree [GP92] so that we can find one standard Young tableau corresponding to one group of fillings. This procedure will give a correspondence satisfying (4.1).

\subsection{Grouping Table}

For the general hook of shape $\mu=\left(s, 1^{n-s}\right)$, the way that we make the grouping table is the following : we start by putting the numbers from 1 to $n$ on the top of the table. We choose $s$ numbers between 1 and $n-(k-1)$ including 1 and $n-(k-1)$, where $k$ changes from 1 to $n-s+1$. Say $1, a_{1}, \ldots, a_{s-2}, n-(k-1)$ are chosen. In the grouping table, we place $\times$ marks under the numbers which are chosen and we place $\circ$ under the unchosen ones. In the next line, we place $\times$ marks under the numbers $2, a_{1}+1, \ldots, a_{s-2}+1, n-(k-1)+1$. We keep making lines with the numbers increasing by 1 at a time until the largest number $n-(k-1)+j$ becomes $n$, after repeating $j$ times. Then this procedure will generate $k$ lines and these $k$ lines are considered as one set which will correspond to one standard Young tableau. We repeat this procedure in all possible $\left(\begin{array}{c}n-k-1 \\ s-2\end{array}\right)$ ways, where $1 \leqslant k \leqslant n-s+1$ and this completes the construction. Table 1 is an example of the grouping table for $\mu=(3,1,1)$, where $n=5, s=3$.

Given one set of $k$ lines in the grouping table, we read out the fillings in the following way. In $\mu=\left(s, 1^{n-s}\right)$, we place the $s$ chosen numbers in the first row and permute in all possible ways, and put the $n-s$ unchosen numbers in the first column above $(1,1)$ cell and permute in all possible ways. Combining the separate fillings for the first row and the first column (not including $(1,1)$ cell) gives one set of fillings. Repeat the same thing with different choices if there are more than one line in one set. From one $k$-lined set, we get $k \cdot s !(n-s)$ ! fillings. For instance, in Table 1 , from the first line

\begin{tabular}{lllll}
1 & 2 & 3 & 4 & 5 \\
\hline$\times$ & $\times$ & $\circ$ & $\circ$ & $\times$ \\
\hline
\end{tabular}

we get 12 different fillings

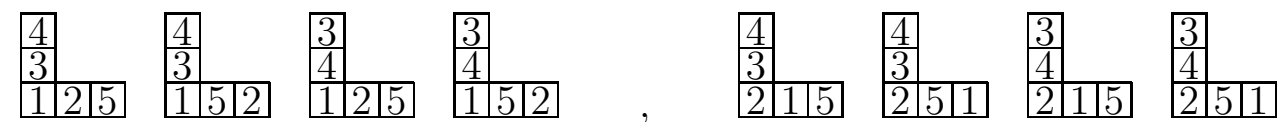




\begin{tabular}{ccccc}
1 & 2 & 3 & 4 & 5 \\
\hline$\times$ & $\times$ & $\circ$ & $\circ$ & $\times$ \\
\hline$\times$ & $\circ$ & $\times$ & $\circ$ & $\times$ \\
\hline$\times$ & $\circ$ & $\circ$ & $\times$ & $\times$ \\
\hline$\times$ & $\times$ & $\circ$ & $\times$ & $\circ$ \\
$\circ$ & $\times$ & $\times$ & $\circ$ & $\times$ \\
\hline$\times$ & $\circ$ & $\times$ & $\times$ & $\circ$ \\
$\circ$ & $\times$ & $\circ$ & $\times$ & $\times$ \\
\hline$\times$ & $\times$ & $\times$ & $\circ$ & $\circ$ \\
$\circ$ & $\times$ & $\times$ & $\times$ & $\circ$ \\
$\circ$ & $\circ$ & $\times$ & $\times$ & $\times$ \\
\hline
\end{tabular}

Table 1: The grouping table for $\mu=(3,1,1)$.

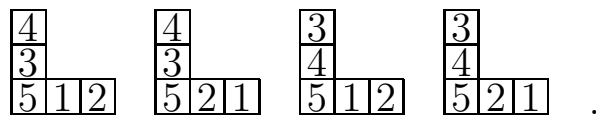

An advantage of having the grouping table is that we do not have to calculate $q^{\operatorname{inv}\left(\mu, \sigma_{i}\right)}$ $\times t^{\operatorname{maj}\left(\mu, \sigma_{i}\right)}$ for all $\sigma_{i}$ 's coming from one set of fillings, for we can read out the polynomial in the right hand side of (4.1) directly from the grouping table. One $k$-lined set would give

$$
[s-1]_{q} ![n-s]_{t} ![k]_{t}\left(\sum_{j=1}^{s} q^{j-1} t^{b\left(r_{j}^{k}\right)}\right),
$$

where $r_{j}^{k}$ 's are the chosen numbers in the last line of the set and $b\left(r_{j}^{k}\right)$ is the number of unchosen numbers which are bigger than $r_{j}^{k}$ (or, number of o's to the right of $r_{j}^{k}$ ). Full explanation for the proof of (4.2) is given in Proposition 4.3. We give the precise proof that this result is exactly the right hand side of (4.1) in Proposition 4.2 and Proposition 4.3 .

\subsection{Modified Garsia-Procesi Tree}

By using the grouping table, we can connect a group of fillings to a polynomial coming from the combinatorial construction in (3.9) corresponding to one standard Young tableau. Namely, we associate a SYT to a group of lines in the grouping table such that (4.2) coincides with the right hand side of (4.1), for the corresponding SYT. However, it does not explicitly determine what the corresponding standard Young tableau is. To specify the described correspondence, we consider the Garsia-Procesi tree [GP92] that was used to find the basis of certain graded $S_{n}$ modules which has a character related to the KostkaFoulkes polynomials $K_{\lambda \mu}(t)$. Garsia and Procesi used their tree to find a basis of the graded $S_{n}$ module, but since we are calculating the Hilbert series, we just recall how we construct the tree to find the Hilbert series. The Young diagram of $\mu$ is the root of the tree, and children are obtained by removing one corner square. We put multiple edges as 
many as the number of squares of arm 0 in the same column of the square to be removed. We keep removing corner squares one at each level until only one square is left. Then we assign appropriate weights on branches of the tree so that we get

$$
\sum_{\lambda \vdash n} \tilde{K}_{\lambda \mu}(t) f_{\lambda}=\sum_{T \in \operatorname{SYT}(\mu)} W(T)
$$

where $\tilde{K}_{\lambda \mu}(t)=\tilde{K}_{\lambda \mu}(0, t)$ are the modified Kostka-Foulkes polynomials, $f_{\lambda}$ is the number of standard Young tableaux of shape $\lambda$, and $W(T)$ is a polynomial which can be defined as follows. Given a partition $\mu$ we assign a weight $w(c)$ to each corner square $c$ according to the following rule. If the coordinates of $c$ are $\left(i, \mu_{i}\right)$ and $m$ is the multiplicity of $\mu_{i}$ in $\mu$ then $w(c)=t^{i-m}+t^{i-m+1}+\cdots t^{i-2}+t^{i-1}$. Finally, for a standard Young tableau $T$ we set $W(T)$ equal to the product of the weights of each of its entries, here the weight of entry $s$ in $T$ is taken to be the weight of the corner square containing $s$ in the partition obtained from the shape of $T$ by removing all the squares containing entries bigger than $s$. We give an example of the Garsia-Procesi tree for $(2,1,1)$ in Figure 2.

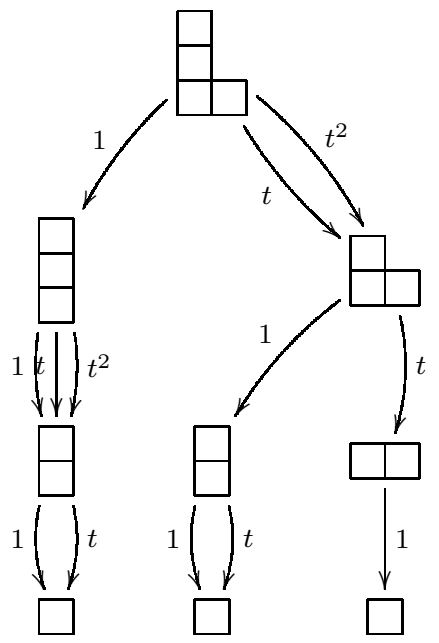

Figure 2: The Garsia-Procesi tree for $\mu=(2,1,1)$.

Then we get the Hilbert series as a sum of the following polynomials

$$
\begin{aligned}
& w\left(\begin{array}{ll}
\hline \frac{3}{2} \\
\hline 1 & 4
\end{array}\right)=\left(1+t+t^{2}\right)(1+t), \\
& 4 \\
& 2 \\
& w\left(\begin{array}{ll}
1 & 3
\end{array}\right)=\left(t+t^{2}\right)(1+t), \\
& 4 \\
& w\left(\begin{array}{ll}
1 & 2
\end{array}\right)=\left(t+t^{2}\right)(t)
\end{aligned}
$$

which is the right hand side of (3.9) when $q=0$. The paths from the leaves can be identified with standard Young tableaux of shape $\mu$. As we trace back the tree, we fill the 
added cell by $i$, where $i$ changes from 1 to $n$. Noting that the Garsia-Procesi tree gives the Hilbert series of $M_{\mu}$ when $q=0$, we modify the tree so that we can recover $q$-statistics.

Given the polynomials of the form $[s-1]_{q} ![n-s]_{t} ![k]_{t}\left(\sum_{j=1}^{s} q^{j-1} t^{b\left(r_{j}\right)}\right)$ from the group of fillings, we modify the Garsia-Procesi tree [GP92] and use it to find the corresponding standard Young tableau. We modify the Garsia-Procesi tree as follows. The way of putting multiple edges are the same to the construction of the original Garsia-Procesi tree. The $k$-multiple edges would have the weight $1, t, \ldots, t^{k-1}$. For the $q$-statistics, in the beginning of the tree, we put 0's above the cells in the first row to the right of the $(1,1)$ cell. As we go down one branch in the tree, if a cell in the first column is removed, then we increase the numbers above the first row by 1 . And if a cell in the first row is removed, the number above that removed cell will be fixed. For the running example of $\mu=(3,1,1)$, the modified Garsia-Procesi tree is given in the Figure 3. In the last leaves

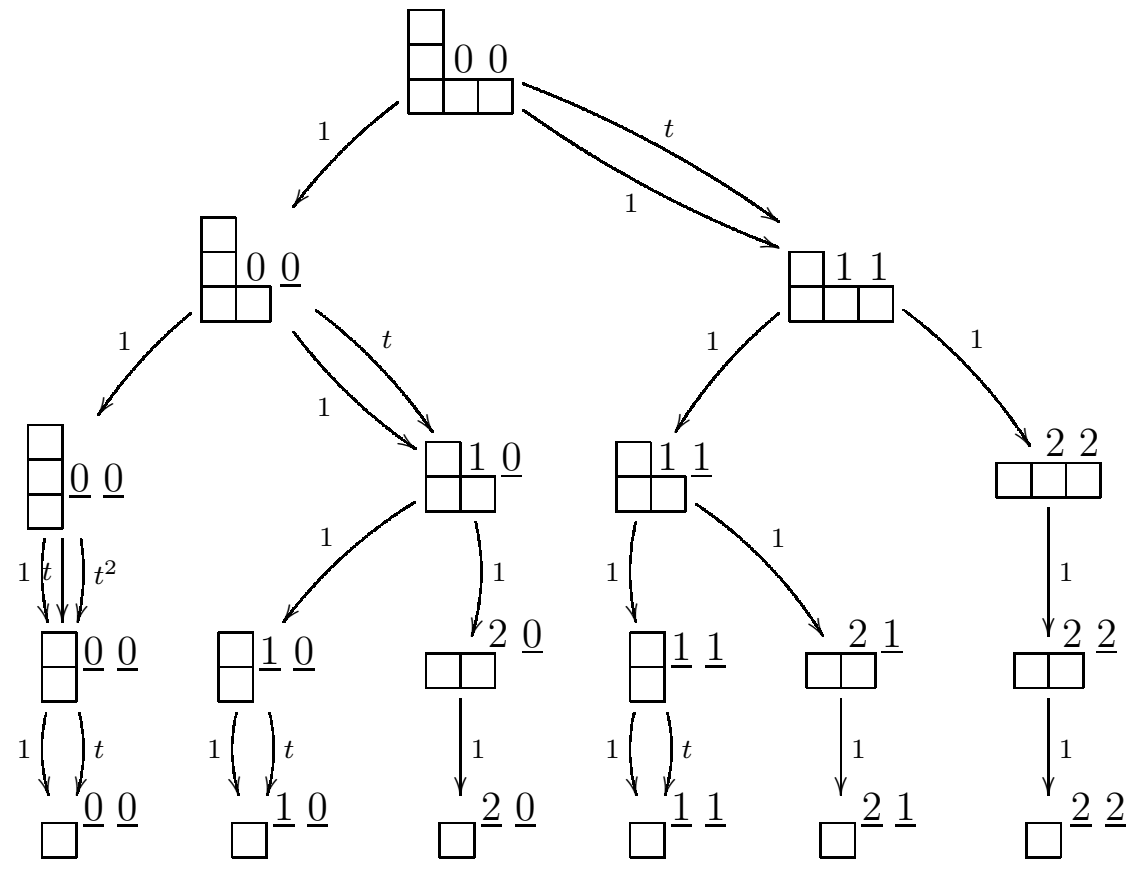

Figure 3: The modified Garsia-Procesi tree for $\mu=(3,1,1)$.

of the tree, we compare the fixed $s-1$ many numbers reading from left to right which were above the first row (to the right of $(1,1)$ cell) to $b\left(r_{1}\right), \ldots, b\left(r_{s-1}\right)$. The leaf having $b\left(r_{1}\right), \ldots, b\left(r_{s-1}\right)$ to the right is the starting point for constructing the standard tableau and we trace back the tree. We put 1 in the starting leaf and follow up the path and put 2 in the added cell. We fill the tableau as we trace back up the tree putting the next element in the added cell. For example, from the first line of the grouping table in Table 1 we get the polynomial

$$
(1+t)(1+q)\left(t^{2}+q t^{2}+q^{2}\right) .
$$

We look for $b\left(r_{1}\right), b\left(r_{2}\right)=2,2$ in the bottom leaves of Figure 3 which is the right end leaf 
and as we trace back the tree, we fill the diagram with numbers from 1 to $n=5$ :

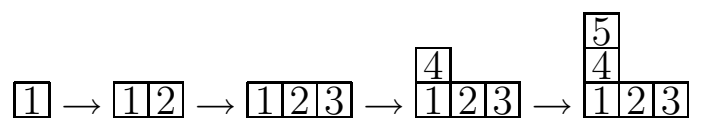

The final tableau is the corresponding standard Young tableau which gives the same polynomial by the combinatorial construction in (3.9).

Remark 4.1. We can find the polynomials for the Hilbert series corresponding to standard Young tableaux by just considering the modified Garsia-Procesi tree. We start from one last leaf in the bottom of the tree. The numbers to the right of the cell (the last leaf in the tree) give $b\left(r_{1}\right), \ldots, b\left(r_{s-1}\right)$ from left to right which makes the factor $\left(\sum_{j=1}^{s-1} q^{j-1} t^{b\left(r_{j}\right)}+q^{s}\right)$. We trace back the tree and pick up the weights on the paths. If there are multiple edges with weights $1, t, \ldots, t^{k}$, then we pick up $\left(1+t+\cdots+t^{k}\right)$ from that path. We multiply the contributions along the path from the bottom to top of the tree and multiply $[s]_{q}$ ! then this gives one polynomial corresponding to one standard Young tableau.

For instance, from the right end path in Figure 3, we get $\left(t^{2}+q t^{2}+q^{2}\right) \cdot 1 \cdot 1 \cdot 1 \cdot(1+t)$ and we multiply $[2]_{t} !=(1+q)$ and get

$$
(1+t)(1+q)\left(t^{2}+q t^{2}+q^{2}\right)
$$

We give the proof that the grouping table and the modified Garsia-Procesi tree give a bijection between sets of fillings and standard Young tableaux of shape $\mu$. We first prove that the grouping table gives the Hilbert series of Garsia-Haiman modules.

Proposition 4.2. The grouping algorithm generates all the $n$ ! fillings of $\mu$.

Proof. By the construction the grouping table, we do not count the same filling multiple times, so we only need to check that the number of fillings that are counted in the grouping table is $n$ !. From the permutations on the first column and the first row not including $(1,1)$ we count $(s-1)$ ! $(n-s)$ !. In the grouping table, there are $\left(\begin{array}{c}n-k-1 \\ s-2\end{array}\right)$ many $k$-lined sets and in each line there are $s$ different choices for the element in the cell $(1,1)$. We add them up to get the number of fillings that the grouping table counts :

$$
s(s-1) !(n-s) ! \sum_{k=1}^{n-s+1} k\left(\begin{array}{c}
n-k-1 \\
s-2
\end{array}\right)=s !(n-s) ! \sum_{k=1}^{n-s+1} k\left(\begin{array}{c}
n-k-1 \\
s-2
\end{array}\right) .
$$

Therefore, we want to show the following identity

$$
\left(\begin{array}{l}
n \\
s
\end{array}\right)=\sum_{k=1}^{n-s+1} k\left(\begin{array}{c}
n-k-1 \\
s-2
\end{array}\right)
$$

Note the known identity

$$
\sum_{j=k}^{n}\left(\begin{array}{l}
j \\
k
\end{array}\right)=\left(\begin{array}{l}
n+1 \\
k+1
\end{array}\right)
$$


Then, by applying (4.4) twice, the right hand side of (4.3) becomes

$$
\begin{aligned}
\sum_{k=1}^{n-s+1} k\left(\begin{array}{c}
n-k-1 \\
s-2
\end{array}\right) & =\sum_{j=1}^{n-s+1} \sum_{k=1}^{n-s+1}\left(\begin{array}{c}
n-k-1 \\
s-2
\end{array}\right) \\
& =\sum_{k=1}^{n-s+1}\left[\sum_{j=s-2}^{n-k-1}\left(\begin{array}{c}
j \\
s-2
\end{array}\right)\right]=\sum_{k=1}^{n-s+1}\left(\begin{array}{c}
n-k \\
s-1
\end{array}\right) \\
& =\sum_{j=s-1}^{n-1}\left(\begin{array}{c}
j \\
s-1
\end{array}\right) \\
& =\left(\begin{array}{c}
n \\
s
\end{array}\right),
\end{aligned}
$$

which is the left hand side of (4.3). This shows that we considered all $n$ ! possible fillings, hence the grouping table gives the Hilbert series of $M_{\mu}$.

In Proposition 4.2 we confirm that the grouping table gives the Hilbert series of $M_{\mu}$. In the following proposition, we show that each $k$-lined set in the grouping table, for $k \leqslant 1 \leqslant n-s+1$, gives the set of fillings corresponding to one standard Young tableau.

Proposition 4.3. The identity (4.1) holds, where the left hand side is determined by the grouping algorithm, and the SYT T in the right hand side is given by the modified Garsia-Procesi tree.

Proof. We show that if we add up all the terms $q^{\operatorname{inv}\left(\mu, \sigma_{i}\right)} t^{\operatorname{maj}\left(\mu, \sigma_{i}\right)}$ for $\sigma_{i}$ 's coming from one $k$-lined set of the grouping table, we get a polynomial of the type $\prod_{i=1}^{n}\left[a_{i}(T)\right]_{t}[s-$ $1]_{q} !\left(\sum_{j=1}^{s-2} q^{j-1} t^{b_{j}(T)}+q^{s-1}\right)$ for a standard Young tableau $T$ of shape $\mu$ where $\mu=$ $\left(s, 1^{n-s}\right)$, which is the right hand side of (4.1).

Recall the way that we get the fillings from one set of the grouping table. First, we place $s$ chosen numbers in the first row in all possible ways, and $n-s$ unchosen numbers in the first column above the $(1,1)$ cell in all possible ways and combine them to get all possible fillings. We note the following fact first.

Lemma 4.4. For a given $n+1$ numbers $\left\{\sigma_{1}, \ldots, \sigma_{k}, k, \sigma_{k+1}, \ldots, \sigma_{n}\right\}, \sigma_{1}<\sigma_{2}<\cdots<$ $\sigma_{k}<k<\sigma_{k+1}<\cdots<\sigma_{n}$, we consider all the possible fillings for one column tableau $\left(1^{n+1}\right)$ with permutations of $\left\{\sigma_{1}, \ldots, \sigma_{k}, \sigma_{k+1}, \ldots, \sigma_{n}\right\}$ fixing $k$ in the bottom $(1,1)$ cell. Then we have

$$
\sum_{\substack{\sigma \in S_{n} \\ k \text { in }(1,1) \text { cell }}} t^{m a j(\sigma)}=t^{n-k}[n]_{t} ! .
$$

Note that $n-k$ is the number of elements which are bigger than $k$ in the $(1,1)$ cell.

Proof. Without considering $k$ in the bottom cell, for the permutations $\sigma \in S_{n}$ of $n$ numbers $\left\{\sigma_{1}, \ldots, \sigma_{k}, \sigma_{k+1}, \ldots, \sigma_{n}\right\}, \sum_{\sigma \in S_{n}} t^{\operatorname{maj}(\sigma)}=[n]_{t}$ ! which is known by Stanley [Sta99, p.364]. We prove (4.5) by induction. If $n=1$, then, If $\sigma_{1}<k$, then it doesn't contribute any maj 
statistic, and if $\sigma_{1}>k$, then it gives 1 to the maj, so $t^{\operatorname{maj}(\sigma)}=t=t[1]_{t}$.

We assume that (4.5) holds for $\mu=\left(1^{n}\right)$. We calculate $\sum_{k \text { in }(1,1) \text { cell }} t^{\operatorname{maj}(\sigma)}$ by varying the number which comes right above $k$ in the bottom cell. If $\sigma_{1}$ is right above $k$, then it does not contribute to maj, and by the induction hypothesis,

$$
\sum_{\substack{\sigma \in S_{n-1, \sigma_{1} \in(2,1)} \\ k \text { in }(1,1) \text { cell }}} t^{\operatorname{maj}(\sigma)}=t^{n-1}[n-1]_{t} ! .
$$

$k$ doesn't contribute any maj if we vary the numbers in the cell $(2,1)$ within $\left\{\sigma_{1}, \ldots, \sigma_{k}\right\}$, and the exponent of $t$ multiplied to $[n-1]_{t}$ ! decreases by 1 as $\sigma_{i}$ increases. Now, if $\sigma_{k+1}$ comes in the cell $(2,1)$, then $\sigma_{k+1}$ and $k$ will make a descent and so $\sigma_{k+1}$ gives $n$ to maj. So,

$$
\sum_{\substack{\sigma \in S_{n-1}, \sigma_{k+1} \in(2,1) \\ k \text { in }(1,1) \text { cell }}} t^{\operatorname{maj}(\sigma)}=t^{2 n-k-1}[n-1]_{t} ! .
$$

As $\sigma_{i}$ changes in $\left\{\sigma_{k+1}, \ldots, \sigma_{n}\right\}, \sigma_{i}$ and $k$ make a descent so $\sigma_{i}$ contributes $n$, and the exponent of $t$ decreases by 1 as $\sigma_{i}$ increases. Finally, we add up all possible cases, and get

$$
\begin{aligned}
\sum_{\substack{\sigma \in S_{n} \\
k \text { in }(1,1) \text { cell }}} t^{\operatorname{maj}(\sigma)} & =[n-1]_{t} !\left(t^{n-1}+\cdots+t^{n-k}+t^{2 n-k-1}+\cdots+t^{n}\right) \\
& =t^{n-k}[n-1]_{t} !\left(1+t+\cdots+t^{k-1}+t^{k}+\cdots+t^{n-1}\right) \\
& =t^{n-k}[n-1]_{t} ![n]_{t}=t^{n-k}[n]_{t} !
\end{aligned}
$$

Since the major and inversion statistics are equidistributed over the symmetric group [Mac13], we have that

$$
\sum_{\sigma \in S_{s-1}} q^{\operatorname{inv}(\sigma)}=[s-1]_{q} !
$$

Thus, the permutations of $s-1$ elements in the first row not including the $(1,1)$ cell give $[s-1]_{q}$ ! factor, and the permutations of $n-s$ elements in the first column above the $(1,1)$ cell give $[n-s]_{t}$ ! factor. So summing up $q^{\operatorname{inv}\left(\mu, \sigma_{i}\right)} t^{\operatorname{maj}\left(\mu, \sigma_{i}\right)}$ for $\sigma_{i}$ 's coming from one line of the grouping table gives $q^{a\left(r_{j}\right)} t^{b\left(r_{j}\right)}[n-s]_{t} ![s-1]_{q}$ !, where $r_{j}$ 's are the chosen numbers, $r_{1}<r_{2}<\cdots<r_{s}$, and $a\left(r_{j}\right)$ and $b\left(r_{j}\right)$ are determined by the element $r_{j}$ in the $(1,1)$ cell as follows. In the grouping table, $a\left(r_{j}\right)$ counts the number of chosen numbers strictly less than $r_{j}$ (or, number of $\times$ 's to the left of $r_{j}$ ) since $r_{j}$ makes an inversion triple with those numbers, and $b\left(r_{j}\right)$ counts the number of unchosen numbers strictly bigger than $r_{j}$ (or, the number of o's to the right of $r_{j}$ ) which is the contribution to maj by $r_{j}$ by Lemma 4.4. Notice that by the way of constructing the $k$-lines sets in the grouping table, all lines in the same set share the factor $\left(\sum_{j=1}^{s} q^{a\left(r_{j}\right)}\right)$ and $b\left(r_{j}\right)^{\prime}$ 's uniformly decrease by 1 as line 
goes down. In other words, if we let $r_{j}^{i}, r_{1}^{i}<\cdots<r_{s}^{i}$, be the chosen numbers in the $i^{\text {th }}$ line in one $k$-lined set, then

$$
\sum_{j=1}^{s} q^{a\left(r_{j}^{p}\right)}=\sum_{j=1}^{s} q^{a\left(r_{j}^{q}\right)}, \quad \text { for any } 1 \leqslant p, q \leqslant k
$$

and

$$
b\left(r_{j}^{p}\right)=1+b\left(r_{j}^{p+1}\right), \quad \text { for } 1 \leqslant p \leqslant k-1,1 \leqslant j \leqslant s .
$$

Also, notice that $a\left(r_{j}^{i}\right)=j-1$ for any $1 \leqslant i \leqslant k$ by the construction of the grouping table. Hence, summing up $q^{\operatorname{inv}\left(\mu, \sigma_{i}\right)} t^{\operatorname{maj}\left(\mu, \sigma_{i}\right)}$ for all $\sigma_{i}$ 's coming from one set of fillings gives (4.2) where the $r_{j}^{k}$ 's, $r_{1}^{k}<\cdots<r_{s}^{k}$, are the chosen numbers in the last line of one $k$-lined set. Since $r_{j}^{k}$ 's are in the last line of one set, $r_{s}^{k}=n$ and this gives $b\left(r_{s}^{k}\right)=0$. So (4.2) is equal to

$$
[n-s]_{t} ![s-1]_{q} ![k]_{t}\left(\sum_{j=1}^{s-1} q^{j-1} t^{b\left(r_{j}^{k}\right)}+q^{s-1}\right) .
$$

Note that there are $\left(\begin{array}{c}n-k-1 \\ s-2\end{array}\right)$ many $k$-lined sets in the grouping table.

Now we find a standard Young tableau which could have (4.6) as a result the combinatorial calculation as defined in (3.9). Given a sequence of $b\left(r_{j}^{k}\right)^{\prime}$ s, $j=1, \ldots, s-1$,

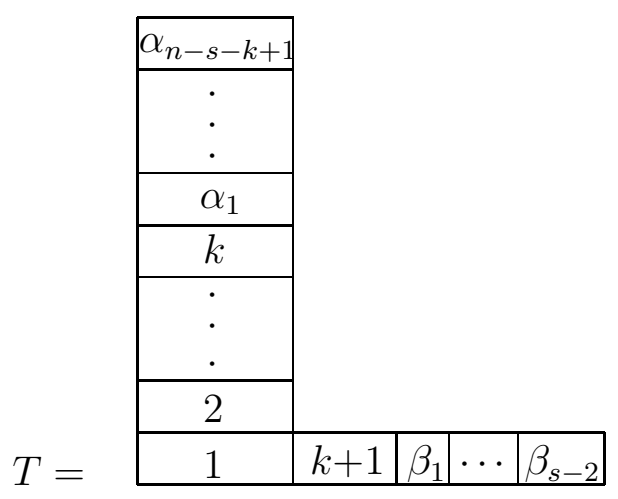

consider a standard Young tableau $T$ of a shape $\mu=\left(s, 1^{n-s}\right)$ with the filling $\alpha_{n-s-k+1}>$ $\cdots>\alpha_{1}>k>k-1>\cdots>2>1<k+1<\cdots<\beta_{1}<\cdots<\beta_{s-2}$, reading from the top to bottom, from left to right in the same row, such that

$$
\begin{aligned}
& b\left(r_{1}^{k}\right)=\left|\left\{\alpha_{i}: \alpha_{i}>k+1,1 \leqslant i \leqslant n-s-k+1\right\}\right|, \\
& b\left(r_{j}^{k}\right)=\left|\left\{\alpha_{i}: \alpha_{i}>\beta_{j}, 1 \leqslant i \leqslant n-s-k+1\right\}\right|, \text { for } 2 \leqslant j \leqslant s-1 .
\end{aligned}
$$

Then by the choice of the filling of $T$,

$$
\prod_{i=1}^{n}\left[a_{i}(T)\right]_{t}=[1]_{t} \times \cdots \times[k]_{t} \times[k]_{t} \times \cdots \times[n-s]_{t}=[n-s]_{t} ![k]_{t},
$$




$$
\sum_{j=1}^{s-1} q^{j-1} t^{b_{j}(T)}=\sum_{j=1}^{s-1} q^{j-1} t^{b\left(r_{j}^{k}\right)}
$$

Hence,

$$
\begin{aligned}
\prod_{i=1}^{n}\left[a_{i}(T)\right]_{t}[s-1]_{q} !\left(\sum_{j=1}^{s-1} q^{j-1} t^{b_{j}(T)}+q^{s-1}\right) & \\
& =[n-s]_{t} ![k]_{t}[s-1]_{q} !\left(\sum_{j=1}^{s-1} q^{j-1} t^{b\left(r_{j}^{k}\right)}+q^{\mu_{1}-1}\right) .
\end{aligned}
$$

Since there are $\left(\begin{array}{c}n-k-1 \\ s-2\end{array}\right)$ choices for the numbers $\left(\alpha_{1}, \ldots, \alpha_{n-s-k+1} ; \beta_{1}, \ldots, \beta_{s-2}\right)$ for the filling of a standard tableau $T$ of shape $\mu=\left(s, 1^{n-s}\right)$, for each $k$-lined set in the grouping table, we can find one and only one standard Young tableau satisfying (4.7). This finishes the proof.

\section{Haglund Basis}

It is worth of noting that we can realize the Haglund basis [ARR08, Corollary 1.7] of $M_{\mu}$ by extending the grouping table. Adin, Remmel and Roichman [ARR08] studied the Garsia-Haiman Modules for hook shapes and found several bases of the modules including the Haglund basis. The construction of the Haglund basis is based on the combinatorial interpretation of Haglund, Haiman and Loehr [HHL05] for the modified Macdonlad polynomials.

The decent set of a permutation $\pi \in S_{n}$ is

$$
\operatorname{Des}(\pi):=\{i: \pi(i)>\pi(i+1)\}
$$

For every integer $1 \leqslant k \leqslant n$ and permutation $\pi \in S_{n}$ define

$$
\begin{gathered}
d_{i}^{(k)}(\pi):= \begin{cases}|\operatorname{Des}(\pi) \cap\{i, \ldots, k-1\}|, & \text { if } 1 \leqslant i<k ; \\
0, & \text { if } i=k ; \\
|\operatorname{Des}(\pi) \cap\{k, \ldots, i-1\}|, & \text { if } k<i \leqslant n .\end{cases} \\
\operatorname{inv}_{i}^{(k)}(\pi):= \begin{cases}\mid j: i<j \leqslant k \text { and } \pi(i)>\pi(j)\} \mid, & \text { if } 1 \leqslant i<k ; \\
0, & \text { if } i=k ; \\
\mid\{j: k \leqslant j<i \text { and } \pi(j)>\pi(i)\} \mid, & \text { if } k<i \leqslant n .\end{cases}
\end{gathered}
$$

Then the $(n-s+1)^{\text {th }}$ Haglund monomial is defined by

$$
c_{\pi}^{(n-s+1)}:=\prod_{i=1}^{n-s} x_{\pi(i)}^{d_{i}^{(n-s+1)}(\pi)} \cdot \prod_{i=n-s+2}^{n} y_{\pi(i)}^{\operatorname{inv}_{i}^{(n-s+1)}(\pi)} .
$$

Theorem 5.1. [ARR08] $\left\{c_{\pi}^{(n-s+1)}: \pi \in S_{n}\right\}$ forms a basis for the Garsia-Haglund module $M_{\left(s, 1^{n-s}\right)}$. 
We extend the grouping table by specifying the element placing in the $(1,1)$ cell to construct the Haglund monomial basis. We consider one line in the grouping table and let $c$ be one of the chosen numbers which comes in the $(1,1)$ cell, and $r_{1}, \ldots, r_{s-1}$, $r_{1}<\cdots<r_{s-1}$, be the rest of the chosen numbers, and $p_{1}, \ldots, p_{n-s}, p_{1}<\cdots<p_{n-s}$, be the unchosen numbers. Then

$$
\begin{aligned}
\left([n-s]_{x_{p_{n-s}}} \cdot[n\right. & \left.-s-1]_{x_{p_{n-s-1}}} \cdots[1]_{x_{p_{1}}} \cdot \prod_{i=1}^{n-s} x_{p_{i}} \chi\left(p_{i}>c\right)\right) \\
& \times\left([s-1]_{y_{r_{1}}} \cdot[s-1]_{y_{r_{2}}} \cdots[1]_{y_{r_{s-1}}} \cdot \prod_{i=1}^{s-1} y_{r_{i}} \chi\left(r_{i}<c\right)\right),
\end{aligned}
$$

where $\chi(Q)=1$ if $Q$ is true and $\chi(Q)=0$ if $Q$ is false, gives $(n-s) !(s-1) !$ monomial basis elements for $M_{\left(s, 1^{n-s}\right)}$ which correspond to $\left\{c_{\pi}^{(n-s+1)}\right\}$ for all $\pi$ 's corresponding to the filings coming from one line of the grouping table. Note that since each line has $s$ choices for the element $c$ in the $(1,1)$ cell, each line of the grouping table gives $(n-s) !(s-1) ! s=(n-s) ! s$ ! monomial elements, and there are $k$ lines on one $k$-lined set, and there are $\left(\begin{array}{c}n-k-1 \\ s-2\end{array}\right)$ many $k$-lines sets, for $1 \leqslant k \leqslant n-s+1$, by the calculation in the proof of Proposition 4.2 we can see that we get $n$ ! monomial basis elements by this construction.

For instance, in the grouping table of $\mu=(3,1,1)$, Table 1 , the first line

\begin{tabular}{lllll}
1 & 2 & 3 & 4 & 5 \\
\hline$\times$ & $\times$ & $\circ$ & $\circ$ & $\times$ \\
\hline
\end{tabular}

gives

$$
\begin{array}{cl}
\left(1+x_{4}\right) x_{3} x_{4} \cdot\left(1+y_{2}\right) & \text { when } 1 \text { comes in the }(1,1) \text { cell, } \\
\left(1+x_{4}\right) x_{3} x_{4} \cdot\left(1+y_{2}\right) y_{1} & \text { when } 2 \text { comes in the }(1,1) \text { cell, and } \\
\left(1+x_{4}\right) \cdot\left(1+y_{2}\right) y_{1} y_{2} & \text { when } 5 \text { comes in the }(1,1) \text { cell. }
\end{array}
$$

We can check that these are consistent with $\left\{c_{\pi}^{(n-s+1)}\right\}$ where $\pi$ 's are the permutations corresponding to the fillings coming from the line $\times \times \circ \circ \times$ of the grouping table. The checking is straightforward since both constructions are based on the same combinatorial formula of Haglund, Haiman and Loehr [HHL05] for the Macdonald polynomials.

\section{Generalization to a general shape $\mu$}

In this section, we are to discuss the challenges of extending the combinatorial construction for the Hilbert series of Garsia-Haiman modules to arbitrary shapes. We recall that in Section 2.3, when $q=0$ the Hilbert series becomes

$$
\tilde{F}_{\mu^{\prime}}(0, t)=\frac{1}{(1-t)^{n}} \sum_{T \in \operatorname{SYT}(\mu)} \varphi_{T}(t) .
$$


and this gives the combinatorial construction of

$$
\tilde{G}_{\mu^{\prime}}(0, t)=\sum_{T \in \mathrm{SYT}\left(\mu^{\prime}\right)} \prod_{i=1}^{n}\left[a_{i}(T)\right]_{t},
$$

when $q=0$. Since (2.2) is true for general shape of $\mu$, by (6.1), the Hilbert series of $M_{\mu}$ for any shape of $\mu$ would have the form in (6.2) when $q=0$.

Hence, the natural thing to ask is whether one can find a similar combinatorial construction for the Hilbert series of $M_{\mu}$ for general $\mu$ 's as a sum over standard Young tableaux of shape $\mu$ which is consistent with (6.1) and (6.2) when $q=0$.

Example 6.1. When $\mu=(3,2)$ (or $\mu^{\prime}=(2,2,1)$ ), the Hilbert series could be expressed as a sum of factorized polynomials in the second column of the Table 2.

\begin{tabular}{|c|c|c|}
\hline SYT & $\left(\mu^{\prime}\right)$ & Hilbert series $\tilde{F}_{\mu^{\prime}}(q, t)$ \\
\hline \begin{tabular}{|l|l|}
5 & \\
2 & 4 \\
1 & 3 \\
\end{tabular} & \begin{tabular}{|l|l|}
4 & \\
3 & 5 \\
1 & 2 \\
\end{tabular} & $(1+t)(1+q)^{2}\left(1+q+q^{2} t\right),(1+t)(1+q)^{2}\left(1+q t+q^{2} t\right)$ \\
\hline \begin{tabular}{|l|l|}
4 & \\
2 & 5 \\
1 & 3 \\
\end{tabular} & \begin{tabular}{|l|l|}
3 & \\
2 & 5 \\
1 & 4 \\
\end{tabular} & $(1+q t)(1+q)\left(1+q+q^{2} t\right),(1+q t)(1+q)\left(1+q t+q^{2} t\right)$ \\
\hline \begin{tabular}{|l|}
5 \\
3 \\
1 \\
\end{tabular} & & $(1+t)^{2}(1+q)^{2}\left(1+q+q^{2}\right)$ \\
\hline
\end{tabular}

Table 2: The Hilbert series for $\mu^{\prime}=(2,2,1)$

The standard Young tableaux in the first column of the Table 2 are the possible standard Young tableaux corresponding to the polynomials in the right hand side, which are consistent with the combinatorial construction $\prod_{i=1}^{n}\left[a_{i}(T)\right]_{t}$, where $T \in \operatorname{SYT}\left(\mu^{\prime}\right)$, as defined in (3.1) when $q=0$. Even though it seems that $\tilde{F}_{(3,2)^{\prime}}(q, t)$ can be expressed as a sum of factorized polynomials over standard Young tableaux of shape $(3,2)^{\prime}=(2,2,1)$, we haven't been successful to find the appropriate $q$-statistics.

\section{References}

[AG09] S. Assaf and A. Garsia, A kicking basis for the two-column Garsia-Haiman modules, arXiv:0905.2333v1 (2009), 14 pages.

[All02] E. E. Allen, Bitableaux bases for some Garsia-Haiman modules and other related modules, Electron. J. Combin. 9 (2002), no. 1, Research Paper 36, 59 pp.

[ARR08] Ron M. Adin, Jeffrey B. Remmel, and Yuval Roichman, The combinatorics of the Garsia-Haiman modules for hook shapes, Electron. J. Combin. 15 (2008), no. 1, Research Paper 38, 42pp.

[Ava00] Jean-Christophe Aval, Monomial bases related to the $n$ ! conjecture, Discrete Math. 224 (2000), no. 1-3, 15-35. 
[GH93] Adriano M. Garsia and Mark Haiman, A graded representation model for Macdonald's polynomials, Proc. Nat. Acad. Sci. U.S.A. 90 (1993), no. 8, 3607-3610.

[GH96] A. M. Garsia and M. Haiman, Some natural bigraded $S_{n}$-modules and $q, t$-Kostka coefficients, Electron. J. Combin. 3 (1996), no. 2, Research Paper 24, 60 pp., The Foata Festschrift.

[GH08] A. M. Garsia and J. Haglund, A new recursion in the theory of Macdonald polynomials, preprint (2008).

[GP92] A. M. Garsia and C. Procesi, On certain graded $S_{n}$-modules and the $q$-Kostka polynomials, Adv. Math. 94 (1992), no. 1, 82-138.

[Hag] J. Haglund, private conversation.

[Hai99] Mark Haiman, Macdonald polynomials and geometry, New perspectives in algebraic combinatorics (Berkeley, CA, 1996-97), Math. Sci. Res. Inst. Publ., vol. 38, Cambridge Univ. Press, Cambridge, 1999, pp. 207-254.

[Hai01] , Hilbert schemes, polygraphs and the Macdonald positivity conjecture, J. Amer. Math. Soc. 14 (2001), no. 4, 941-1006.

[HHL05] J. Haglund, M. Haiman, and N. Loehr, A combinatorial formula for Macdonald polynomials, J. Amer. Math. Soc. 18 (2005), no. 3, 735-761.

[Mac13] P. A. MacMahon, The Indices of Permutations and the Derivation Therefrom of Functions of a Single Variable Associated with the Permutations of any Assemblage of Objects, Amer. J. Math. 35 (1913), no. 3, 281-322.

[Mac88] I. G. Macdonald, A new class of symmetric functions, Actes du 20e Seminaire Lotharingien 372 (1988), 131-171.

[Mac95] _ Symmetric functions and Hall polynomials, second ed., Oxford Mathematical Monographs, The Clarendon Press Oxford University Press, New York, 1995, With contributions by A. Zelevinsky, Oxford Science Publications.

[Mor08] Hideaki Morita, Garsia-Haiman modules for hook partitions and Green polynomials with two variables, J. Algebra 319 (2008), no. 1, 235-247. MR MR2378068 (2008k:20023)

[Sta99] Richard P. Stanley, Enumerative combinatorics. Vol. 2, Cambridge Studies in Advanced Mathematics, vol. 62, Cambridge University Press, Cambridge, 1999, With a foreword by Gian-Carlo Rota and appendix 1 by Sergey Fomin.

[Ste94] John R. Stembridge, Some particular entries of the two-parameter Kostka matrix, Proc. Amer. Math. Soc. 121 (1994), no. 2, 367-373. 